\title{
RELACIÓN ENTRE TIPOS DE CÁNCER Y ZONAS DE EXPLOTACIÓN PETROLERA EN LA AMAZONIA ECUATORIANA
}

\author{
Autores: \\ René Buitrón Andrade. MD. MSc. $\mathrm{PhD}(\mathrm{c})^{1}$ \\ Patricio Romero Proaño. MSc. ${ }^{1}$
}





\section{Resumen}

El objetivo de la investigación fue ubicar espacialmente los casos de cáncer y/o tumores que son atendidos en el Hospital Oncológico de Especialidad en el lugar de donde procede en el Oriente ecuatoriano y su relación con la presencia de explotación petrolera.

Se llevó a cabo un estudio de correlación ecológica para establecer la asociación existente entre los diferentes tipos de cáncer y/o tumor maligno y el residir en zonas de explotación petrolera. Se incluyeron a todos los casos que fueron diagnosticados de tumor maligno o cáncer y que son residentes en las provincias de Sucumbíos, Orellana, Napo, Pastaza y Zamora Chinchipe y que fueron atendidos en SOLCA (Quito) a partir del 1 de enero del 2002, hasta el 31 de diciembre del 2008.

La información se recopiló del formulario del Registro Nacional de Tumores utilizando la clasificación internacional CIE-10. A los casos de cáncer de la base de datos que procedían de las provincias de Sucumbíos, Orellana, Napo, Pastaza y Zamora Chinchipe se procedió a la georeferenciación del lugar de residencia para luego asociar estas ubicaciones con los puntos también georeferenciados correspondientes a la ubicación de los pozos de explotación petrolera. Con esta información se estableció la relación existente entre el padecimiento de determinados tipos de cáncer y/o tumor maligno y la residencia en zonas de explotación petrolera.

Para el análisis se utilizó el paquete informático SPSS versión 17.0 para Windows. Para el análisis descriptivo se utilizaron medidas de tendencia central y de dispersión, así como tasas de incidencia acumulada. Para el análisis inferencial se utilizaron: ANOVA, regresión múltiple, coeficiente de correlación con sus respectivos intervalos de confianza y el coeficiente de autocorrelación espacial de Morán.

Se encontró que las provincias de mayor prevalencia de cáncer son: Napo (9.3\%) y Sucumbíos (10.8\%). Con riesgo intermedio están Pastaza (5.6\%) y Orellana (5.2\%). Los tipos de cáncer detectados: Tracto Genito urinario femenino (34\%), seguido de cáncer Gástrico e intestinal (16.9\%), cáncer de Piel y relacionados (12.4\%) fueron los de más alta frecuencia.

Palabras clave: Cáncer, incidencia, explotación petrolera. 


\section{Abstrac}

The research aimed to spatially locate cases of cancer and/or tumors that are cared for in Hospital Oncology of specialty in the place where item is in the Ecuadorian Orient and its relationship with the presence of petroleum exploitation.

Carried out a study of ecological correlation to establish the association between the different types of cancer or malignant tumor and reside in areas of petroleum exploitation. We included all cases that were diagnosed malignant tumor or cancer and who are resident in the provinces of Sucumbíos, Orellana, Napo, Pastaza and Zamora-Chinchipe and were treated in SOLCA (Quito) from January 1, 2002, until December 31, 2008.

The information was collected in the form of the national registry of tumors using the international classification ICD-10. Cases of cancer of the database from the provinces of Sucumbíos, Napo, Orellana, Pastaza and Zamora-Chinchipe proceeded to the georeferencing of the place of residence then associate these locations with the points also georeferenced corresponding to the location of oil wells. With this information, was established the relationship between the condition of certain types of cancer or malignant tumor and residence in oil exploitation areas.

The analysis was used for the software package SPSS version 17.0 for Windows. Measures of central tendency and dispersion, as well as cumulative incidence rates were used for descriptive analysis. Used for inferential analysis: ANOVA, multiple regression, coefficient of correlation with their respective confidence intervals and the coefficient of Spatial autocorrelation of Morán.

Found that the provinces of greater prevalence of cancer are: Napo (9.3\%) and Sucumbíos (10.8\%). With intermediate risk are Pastaza (5.6\%) and Orellana (5.2\%). The types of cancer detected: tract genital, female urinary (34\%), followed by gastric cancer and intestinal (16.9\%), skin cancer and related (12.4\%) were the highest frequency. 
Introducción:

Los términos hidrocarburos totales de petróleo (abreviados TPH en inglés) se usan para describir una gran familia de varios cientos de compuestos químicos originados de petróleo crudo. Algunas sustancias químicas que pueden encontrarse en los TPH incluyen a hexano, combustibles de aviones de reacción, aceites minerales, benceno, tolueno, xilenos, naftalina, y fluoreno, como también otros productos de petróleo y componentes de gasolina. Sin embargo, es probable que muestras de TPH contengan solamente algunas, o una mezcla de estas sustancias químicas.

El benceno, conocido también como benzol, es un líquido incoloro de olor dulce, se evapora al aire rápidamente y es sólo ligeramente soluble en agua, siendo además sumamente inflamable. Se produce la exposición al benceno a través de alimentos, bebidas alcohólicas o agua embotellada que es menor que la exposición a través del aire, el agua potable por su parte contiene menos de 0.1 ppm de benceno(1). La fuga de gasolina desde tanques subterráneos, o vertederos de desechos peligrosos que contienen benceno puede contaminar el agua de consumo humano.

La exposición breve ( 5 a 10 minutos) a niveles muy altos de benceno en el aire (10,000 a 20,000 ppm) puede producir la muerte; en tanto que niveles más bajos (700 a 3,000 ppm) pueden producir letargo, mareo, aceleración taquicardia, cefalea, temblores, confusión y pérdida del conocimiento. En la mayoría de los casos, los efectos desaparecerán cuando la exposición termina y la persona empieza a respirar aire fresco. (1)

Los derivados bencénicos, producto de los procesos de refinación del petróleo como los presentes en el humo del tabaco, se han relacionado con una gran variedad de tumores de tipo hematológico, en especial leucemias y linfomas (1-2); los mecanismos de daño al parecer se relacionan con alteraciones de tipo cromosómico sumado a inmunotoxicidad. (3)
Desde el descubrimiento en el Oriente ecuatoriano, éste pasó a constituirse en la principal fuente de ingresos económicos para el país, y dependiendo de los gobiernos los ingresos provenientes de su venta se dedicaron mayoritariamente al pago de la duda externa, sin embargo, para el año 2010, según el Ministerio de Finanzas estos ingresos constituyen cerca de18\% del presupuesto general del Estado. (Fuente: Ministerio de Finanzas y Economía del Ecuador en www. finanzas.gob.ec/pls/portal/url/.../781C4898C170817CE040007F010022 02).

Las operaciones petroleras han tenido un alto costo humano y ambiental, dañando los recursos naturales de los cuales las comunidades indígenas y los colonos dependen para subsistir. La mayor parte del crudo del país proviene del bosque lluvioso amazónico, donde la biodiversidad y los pueblos tradicionales enfrentan constantes amenazas de los impactos de las operaciones petroleras.

El Ecuador cuenta al momento con una capacidad de procesamiento de petróleo de 157.500 barriles por día. Los productos más importantes son la gasolina y el diesel, combustibles de uso mayoritario en el transporte. El sector de hidrocarburos aporta con el $71 \%$ del requerimiento nacional de energía, repartiéndose el porcentaje restante entre fuentes de la biomasa e hidroeléctrica. (4)

En el año 1967 la compañía petrolera Texaco perforó el primer pozo comercial en la Amazonía, para en los siguientes años, llevarse a cabo mayores obras de infraestructura petrolera como fueron el Sistema de Oleoducto Trans Ecuatoriano, (SOTE), y la Vía Coca. Hasta el año 1990 Texaco extrajo el 88\% del total de la producción nacional de petróleo y operó el oleoducto. Se estima que perforó 399 pozos y construyó 22 estaciones de perforación.(4)

La contaminación ambiental en la zona ha sido de variada forma, así por ejemplo, los desechos de crudo, regados regularmente por las carreteras con fines de mantenimiento y control del polvo, 
son esparcidos por el medio ambiente con las lluvias. Por otra parte, se estima que las roturas accidentales en el Sistema del Oleoducto Trans-Ecuatoriano han provocado el derrame de unos 400.000 barriles (16,8 millones de galones) de petróleo. Estas prácticas han contaminado un sinnúmero de ríos, riachuelos y esteros; muchas veces las únicas fuentes de agua para los habitantes de la región. Así mismo, la continua quema de petróleo y de millones de pies cúbicos de gas diariamente producen compuestos altamente tóxicos que contribuyen a la contaminación del aire. (4)

De lo anterior es fácil colegir que como efectos negativos de la explotación petrolera que selleva a cabo en el Ecuador se ha provocado la eliminación al medio ambiente, sea al aire o contaminando el agua de los ríos que abastecen del líquido a los pobladores de la zona, de millones de galones de sustancias tóxicas provenientes petróleo o en forma de gas, evidenciándose niveles de contaminación en las comunidades cercanas a los pozos petroleros por muy encima de los niveles permitidos. Esto fue publicado en el estudio realizado por Miguel San Sebastián y Anna-Karin Hurting, en el año 2002, siendo uno de los objetivos de esta investigación el conocer la incidencia de tumores malignos en el área amazónica y su distribución según edad y género. Las conclusiones son preocupantes, así: el riesgo de padecer cáncer de laringe es allí 30 veces mayor que en otras zonas del país; el cáncer de vías biliares 18 veces mayor, el de hígado y piel 15 veces más y el de estómago 5 veces mayor que en otras provincias.

El estudio se basó en el análisis del agua de los ríos y de los efectos cancerígenos de los componentes del petróleo, además del examen de las poblaciones afectadas y de la investigación estadística del crecimiento de la incidencia de cáncer respecto del aumento de explotación en los últimos 30 años. Se atribuyó la presencia de estos tumores cancerígenos a la presencia de sustancias químicas en el agua básicamente que consumían las comunidades indígenas y los colonos que han llegado a la zona desde hace varios años. Los ríos habitualmente utilizados por los habitantes del lugar están contaminados con petróleo en una proporción 200 y 300 veces mayor al límite permitido para el agua de consumo humano; esta contaminación se produce por la filtración de parte de los 4000 metros cúbicos de deshechos por cada pozo perforado, los cuales son depositados en piscinas excavadas en la tierra. (5)

Aunque se han realizado algunos estudios de impacto de los procesos de explotación petrolera en el Oriente ecuatoriano y su efecto sobre las comunidades aledañas, estos estudios epidemiológicos son aislados y sin suficiente sustento técnico científico como el de San Sebastián et al. (2001), a pesar de lo cual se ha determinado un incremento del riesgo relativo de diferentes tipos de cáncer en poblaciones cercanas a sitios de explotación petrolera. Además del estudio antes mencionado, existen unos pocos estudios que presentan alguna evidencia de estos problemas; sin embargo han sido cuestionados debido a la carencia de rigor científico.

En el presente estudio se pretende ubicar espacialmente la zona de residencia de los pacientes que han sido diagnosticados de cualquier tipo de cáncer o tumor y que son atendidos en el Hospital Oncológico de Especialidad, que proceden de las provincias amazónicas en donde se lleva a cabo la explotación petrolera.

\section{Objetivos}

- Ubicar espacialmente los casos de cáncer y/o tumores que son atendidos en el Hospital Oncológico de Especialidad en el lugar de donde procede en el Oriente ecuatoriano y su relación con la presencia de explotación petrolera.

- Relacionar los casos de cáncer y/o tumores malignos residentes en las provincias de Sucumbíos, Orellana, Napo, Pastaza y Zamora Chinchipe con la proximidad a las zonas de explotación petrolera. 


\section{Metodología}

\section{Tipo de estudio:}

Se llevó a cabo un estudio de correlación ecológica para establecer la asociación existente entre los diferentes tipos de cáncer y/o tumor maligno y el residir en zonas de explotación petrolera.

\section{Criterios de muestra y muestreo:}

Para fines de esta investigación se incluyeron a todos los casos que fueron diagnosticados de tumor maligno o cáncer y que son residentes en las provincias de Sucumbíos, Orellana, Napo y Pastaza. Los casos que se incluyeron en este estudio fueron todos los que acudieron a consulta a SOLCA (Quito) a partir del 1 de enero del año 2002, hasta el 31 de diciembre del año 2008.

\section{Materiales y métodos:}

La investigación se llevó a cabo sobre la información de la base de datos del Hospital de SOLCA (Sociedad de Lucha contra el Cáncer) de la ciudad de Quito. La fuente de información fue el formulario del Registro Nacional de Tumores donde consta cada historia clínica de pacientes tratados en esta unidad de salud. La clasificación de los casos de cáncer y/o tumor se realizó utilizando la clasificación internacional CIE-10.

Se consideró como residentes a las personas que han vivido o continúan viviendo en estas zonas por un lapso de al menos 5 años, antes del diagnóstico de cáncer y/o tumor. Se estableció 5 años de residencia como medida aproximada de exposición crónica. Previo a la obtención y/o confirmación de la información se aplicó o solicitó, si es el caso, el consentimiento informado diseñado para el efecto.

A la base de datos de casos de cáncer procedentes/ residentes de las provincias de Sucumbíos, Orellana, Napo y Pastaza se procedió a georeferenciar, es decir a ubicar de acuerdo a su lugar de residencia declarado en una ubicación espacial exacta en las parroquias de sus correspondientes cantones, para luego asociar estas ubicaciones con los puntos también georefenciados correspondientes a la ubicación de los pozos de explotación petrolera. Con esta información se estableció la relación existente entre el padecimiento de determinados tipos de cáncer y/o tumor maligno y la residencia en zonas de explotación petrolera.

Las fuentes de recolección de información y los mecanismos de obtención de la misma se presentan en el siguiente diagrama:

\section{Obtención de la información}

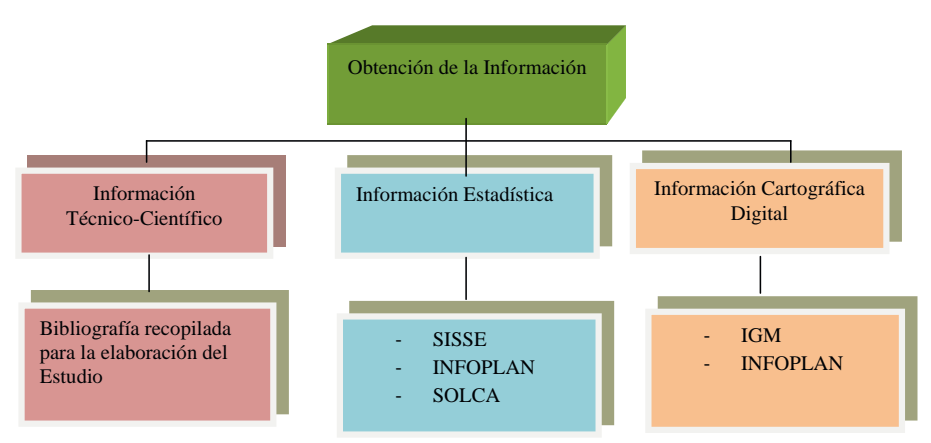

\section{Homogenización de la información}

Una vez revisada la información se procedió a la homogenización de la misma, para lo cual se definió la proyección de coordenadas y presentación de la información con las siguientes especificaciones técnicas:

\begin{tabular}{|l|l|}
\hline Proyección & Universal Transversa de M ercator \\
\hline Elipsoide & Internacional \\
\hline Datum & W GS84 \\
\hline Zona & 18 \\
\hline
\end{tabular}

\section{Procedimiento}

Una vez homogenizada la información cartográfica se realizó la identificación de los bloques petroleros ubicados en la región amazónica ecuatoriana, con su respectivo estatus operacional, con el fin de realizar un traslape con las coberturas provinciales y poder 
medir el área de los bloques petroleros dentro de cada provincia de la región.

La representación cartográfica se realizó en el programa ARCGis

\section{Plan de análisis:}

Con la información recogida en campo se procedió al respectivo análisis estadístico utilizando el paquete informático SPSS versión 17.0 para Windows, con licencia de la Universidad Tecnológica Equinoccial.

El análisis se lo llevó a cabo de la siguiente manera:

Para el análisis descriptivo se utilizaron medidas de tendencia central y de dispersión, así como tasas de incidencia acumulada.

Para el análisis inferencial se utilizaron: regresión múltiple, coeficiente de correlación y el coeficiente de autocorrelación espacial de Morán.

\section{RESULTADOS GENERALES:}

El presente estudio intentó establecer geográficamente la ubicación de las personas afectadas por cualquier tipo de neoplasia y/o tumor maligno, que fueron atendidas en el Hospital de SOLCA de la ciudad de Quito y que se registró su procedencia de alguna de las provincias del Oriente ecuatoriano consideradas, esto es: Sucumbíos, Orellana, Napo y Pastaza.

Para este fin, se tomó como base a los datos disponibles en el Registro Nacional de Tumores de SOLCA. Posteriormente a la recopilación y depuración de la información se georeferenció el lugar de residencia a nivel cantonal en las respectivas provincias; $\sin$ embargo, lamentablemente en muchos casos el lugar de residencia correspondía al lugar en donde los pacientes se encontraban viviendo en ese momento; es decir, la dirección en la ciudad de Quito, en donde temporalmente residían previo a acudir al hospital, y en otros casos esta información no se encontró disponible.

Se obtuvo información oncológicamente válida para el análisis de 471 casos que acudieron a buscar atención en SOLCA en el período comprendido entre el 1 de enero del año 2002 y el 31 de diciembre del año 2008.

Con esta información se procedió a ubicar los casos espacialmente, para luego caracterizar el perfil demográfico de los pacientes, estableciéndose además las tasas de incidencia acumulada de las diversas patologías oncológicas reportadas tanto por provincias como por los cantones que las conforman, cuya distribución se presenta a continuación:

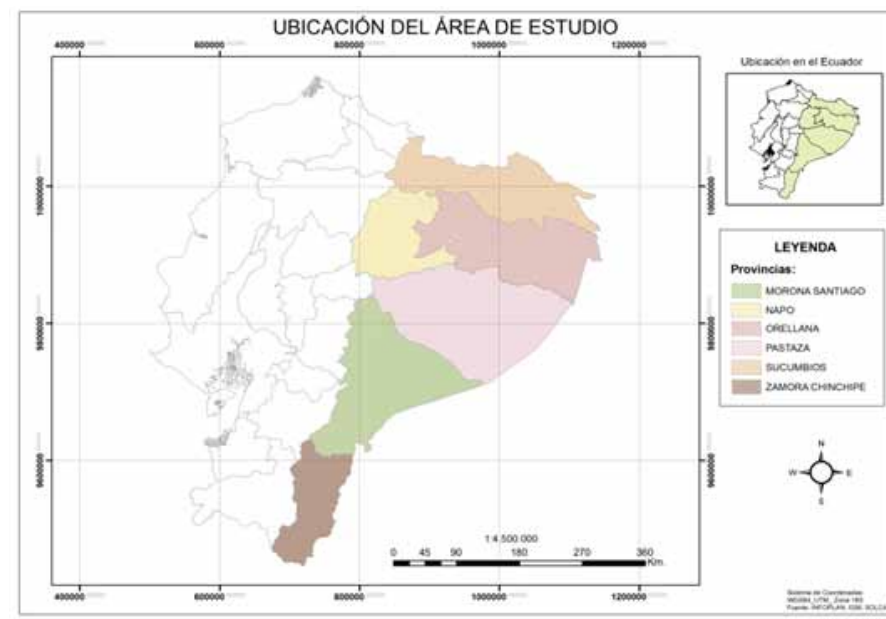

GRÁFICO \# 1

DISTRIBUCIÓN GEOGRÁFICA DEL CÁNCER EN LAS PROVINCIAS DEL ORIENTE ECUATORIANO

Fuente: IGM

Elaboración: Autores

Se han reportado casos de cáncer en todas las provincias del Oriente, y de acuerdo a la base de datos obtenida, según el lugar de residencia de los pacientes que acudieron a SOLCA, se estableció que 
de los 41 cantones en total que conforman estas provincias, entre los años 2002 y 2008 , en $68 \%$ de los cantones se ha reportado al menos un caso de cáncer. Las provincias en las que todos los cantones han reportado al menos un paciente con cáncer son: Sucumbíos, Orellana, Napo y Pastaza.

\section{CARACTERÍSTICAS DEMOGRÁFICAS DE LOS CASOS DE} CÁNCER QUE ACUDEN A SOLCA QUITO, PROCEDENTES DE LA ZONA

\section{DE ESTUDIO:}

Se estudiaron un total de 471 pacientes, cuyo promedio de edad fue de 51 años, con una moda de 50 años y una mediana de 51 años. La edad mínima de los pacientes fue de 2 años en tanto que su máxima edad fue de 88 años. La distribución de las edades por percentiles 25, 50 y 75, cuyas edades corresponden a 38,51 y 62 años, se aprecia en el siguiente boxplot:

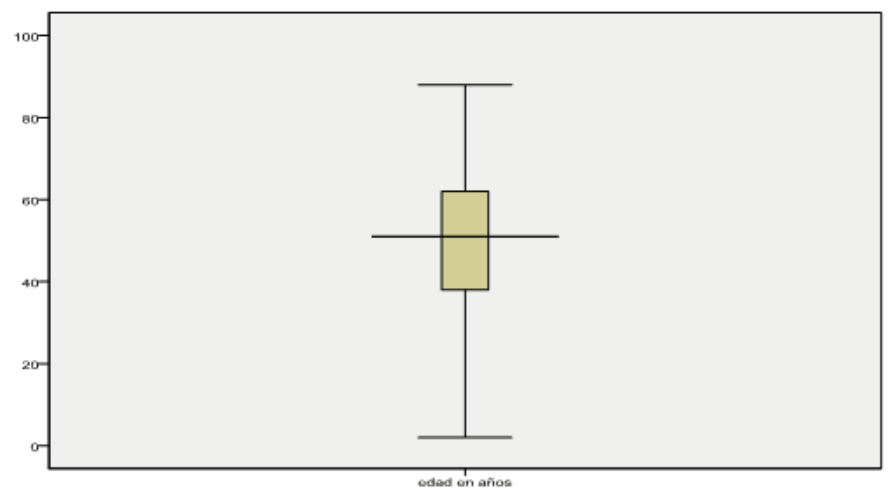

GRÁFICO \# 2

BOX PLOT DE LA DISTRIBUCIÓN PORCENTUAL DE LAS EDADES DE LOS PACIENTES ATENTIDOS EN SOLCA QUITO PROCEDENTES DEL ORIENTE ECUATORIANO, ENTRE LOS AÑOS 2002 Y 2008

Fuente: SOLCA

Elaboración: Autores.

No se evidenciaron valores aberrantes en la variable edad.
El $34,4 \%$ de los pacientes que son atendidos en SOLCA corresponden al género masculino; en tanto que, el restante porcentaje son mujeres. Durante este período, del 22,7\% de los pacientes atendidos han fallecido.

PRESENTACIÓN DE LAS CASOS CON DIAGNÓSTICO DE

\section{CÁNCER Y/O TUMOR:}

La localización de las neoplasias durante el periodo de tiempo de estudio fue muy variada, por lo que se procedió a agruparlas en aparatos y sistemas como se observa en la tabla número 1:

\begin{tabular}{|l|c|c|c|c|c|c|}
\hline \multicolumn{1}{|c|}{ Tipo de Cáncer } & Hombres & $\%$ & Mujeres & $\%$ & $\begin{array}{c}\text { Total } \\
\text { general }\end{array}$ & $\%$ general \\
\hline $\begin{array}{l}\text { Aparato Genital } \\
\text { Masculino }\end{array}$ & 26 & 17,8 & & 0,0 & 26 & 6,5 \\
\hline Cavidad Oral & 8 & 5,5 & 5 & 1,9 & 13 & 3,2 \\
\hline Desconocido & 3 & 2,1 & 3 & 1,2 & 6 & 1,5 \\
\hline Glándula Mamaria & 1 & 0,7 & 30 & 11,7 & 31 & 7,7 \\
\hline Piel y Relacionados & 27 & 18,5 & 23 & 8,9 & 50 & 12,4 \\
\hline $\begin{array}{l}\text { Sistema } \\
\text { Hematopoyético }\end{array}$ & 21 & 14,4 & 9 & 3,5 & 30 & 7,4 \\
\hline Sistema Nervioso & 5 & 3,4 & 4 & 1,6 & 9 & 2,2 \\
\hline Sistema Osteomuscular & 1 & 0,7 & 1 & 0,4 & 2 & 0,5 \\
\hline Sistema Respiratorio & 7 & 4,8 & 3 & 1,2 & 10 & 2,5 \\
\hline Sistema Urinario & 5 & 3,4 & 3 & 1,2 & 8 & 2,0 \\
\hline Tiroides & 1 & 0,7 & 12 & 4,7 & 13 & 3,2 \\
\hline Tracto Gastrointestinal & 41 & 28,1 & 27 & 10,5 & 68 & 16,9 \\
\hline $\begin{array}{l}\text { Tracto Genitourinario } \\
\text { Femenino }\end{array}$ & & 0,0 & 137 & 53,3 & 137 & 34,0 \\
\hline Total general & $\mathbf{1 4 6}$ & $\mathbf{1 0 0 , 0}$ & $\mathbf{2 5 7}$ & $\mathbf{1 0 0 , 0}$ & $\mathbf{4 0 3}$ & $\mathbf{1 0 0 , 0}$ \\
\hline
\end{tabular}

TABLA \# 1

DISTRIBUCIÓN PROPORCIONAL POR APARATOS Y SISTEMAS DE LA PRESENTACIÓN DEL CÁNCER EN LAS PROVINCIAS DEL ORIENTE ECUATORIANO. ECUADOR $2002-2008$

Fuente: SOLCA

Elaboración: Autores.

Como se puede apreciar, en las mujeres el principal cáncer diagnosticado corresponde al aparato genitourinario, con más de la mitad de las neoplasias detectadas, seguido del cáncer de mama y luego del de piel y relacionados. En el caso de los varones en primer lugar se encuentra el cáncer gastrointestinal, seguido del cáncer de piel y relacionados y luego el correspondiente al aparato genital masculino. 
En relación a la edad de los pacientes que acuden a SOLCA Quito, y la localización de su neoplasia, se encontró que en los niños y adolescentes predominan los problemas relacionados con el sistema hematopoyético, en los adultos (comprendidos hasta los 59 años) las neoplasias del tracto genitourinario y en las personas mayores de 60 años, los problemas genitourinarios y gastrointestinales como se aprecia en la siguiente tabla. $(p<0.05)$

\section{TABLA \# 2}

RELACIÓN ENTRE LA EDAD DE LOS PACIENTES PROCEDENTES DEL ORIENTE ECUATORIANO Y LA LOCALIZACIÓN POR APARATOS O SISTEMAS DE LAS NEOPLASIAS $2002-2008$

\begin{tabular}{|l|c|c|c|c|c|}
\hline \multirow{2}{*}{ Sitio definido por sistemas } & \multicolumn{4}{|c|}{ edad recodificada por grupos } & \multirow{2}{*}{} \\
\cline { 2 - 5 } & Niñez & Adolescente & Adulto & Mayor & Total \\
\hline Aparato Genital Masculino & 0 & 1 & 10 & 15 & 26 \\
Cavidad Oral & 0 & 0 & 9 & 4 & 13 \\
Glándula Mamaria & 0 & 0 & 22 & 9 & 31 \\
Piel y Relacionados & 1 & 2 & 23 & 24 & 50 \\
Sistema Hematopoyético & 5 & 6 & 12 & 7 & 30 \\
Sistema Nervioso & 2 & 1 & 6 & 0 & 9 \\
Sistema Respiratorio & 0 & 0 & 6 & 4 & 10 \\
Sistema Urinario & 2 & 0 & 1 & 0 & 3 \\
Sistema Osteo-muscular & 0 & 0 & 2 & 0 & 0 \\
Desconocido & 0 & 0 & 3 & 3 & 6 \\
Tiroides & 0 & 0 & 11 & 2 & 13 \\
Tracto Gastrointestinal & 0 & 0 & 40 & 28 & 68 \\
Tracto Genitourinario & 0 & 0 & 108 & 29 & 137 \\
Total & 10 & 10 & 254 & 129 & 403 \\
\hline
\end{tabular}

Fuente: SOLCA

Elaboración: Autores.

\section{TENDENCIA DE LAS NEOPLASIAS EN LA ZONA DE ESTUDIO:}

Como se mencionó anteriormente, se estableció como periodo de estudio a los años 2002 - 2008, ya que este periodo de tiempo es el que se dispone en la base de datos electrónica de SOLCA. Globalmente la tendencia de la tasa de incidencia acumulada es a una reducción como se puede apreciar en el siguiente gráfico:

\section{TASA DE INCIDENCIA ACUMULADA DE CANCER EN LAS PROVINCIAS DEL}

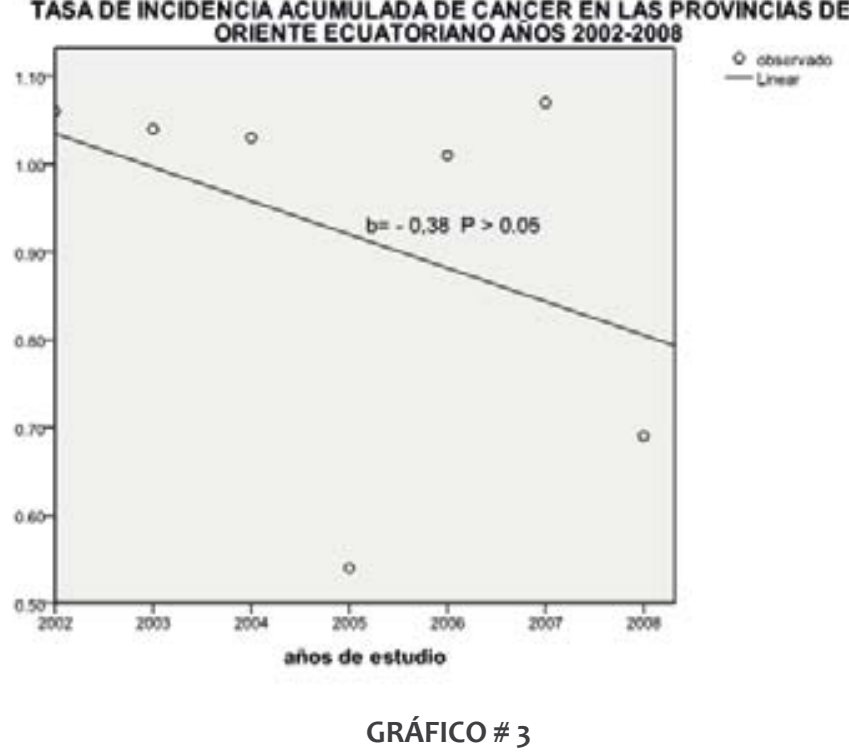

Fuente: SOLCA

Elaboración: Autores

Sin embargo, se puede evidenciar que no hay diferencias significativas que avalen esta posible reducción de la tasa a lo largo del tiempo.

Al analizar por las diferentes provincias del Oriente ecuatoriano, tal como se mencionó antes, existe un gradiente diferencial de presentación de la tasa de incidencia acumulada de cáncer.

La provincia con mayor tasa de incidencia acumulada es Sucumbíos; en ella como se puede apreciar a continuación la tendencia marca también un decrecimiento $(p>0.05)$ 


\section{GRÁFICO \# 4}

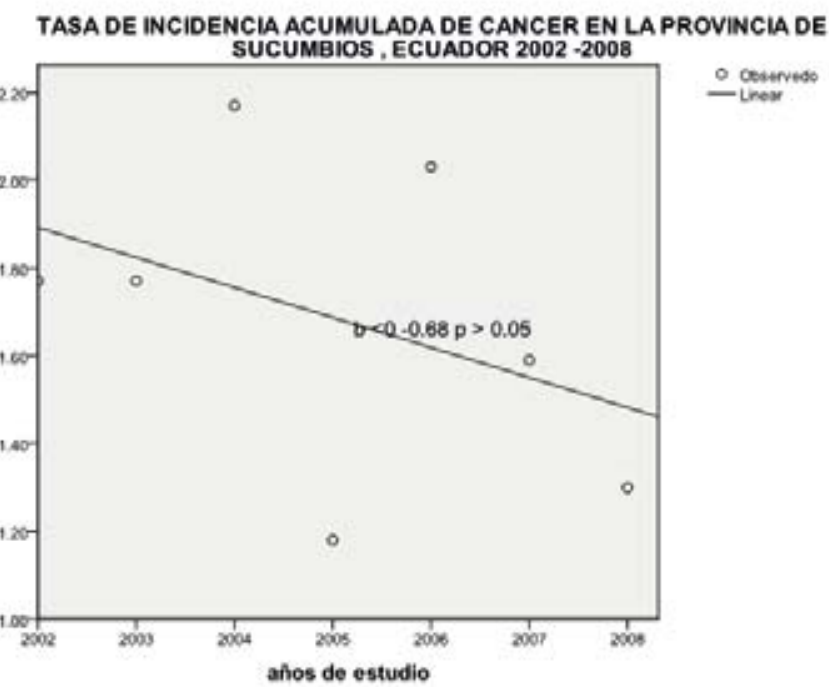

Fuente: SOLCA

Elaboración: Autores

Al interior de esta provincia la totalidad de sus cantones han reportado al menos un caso, siendo su tasa de incidencia variable en los mismos, sin una clara tendencia.

TABLA \# 3

TASA DE INCIDENCIA ACUMULADA DE CÁNCER POR CANTONES EN LA PROVINCIA DE SUCUMBIOS, ECUADOR 2002 - 2008

\begin{tabular}{|c|c|c|c|c|c|c|c|}
\hline CANTONES & $\mathbf{2 0 0 2}$ & $\mathbf{2 0 0 3}$ & $\mathbf{2 0 0 4}$ & $\mathbf{2 0 0 5}$ & $\mathbf{2 0 0 6}$ & $\mathbf{2 0 0 7}$ & $\mathbf{2 0 0 8}$ \\
\hline SUCUMBIOS & $\mathbf{1 , 7 7}$ & $\mathbf{1 , 7 7}$ & $\mathbf{2 , 1 7}$ & $\mathbf{1 , 1 8}$ & $\mathbf{2 0 3}$ & $\mathbf{1 , 5 9}$ & $\mathbf{1 , 3 0}$ \\
\hline CASCALES & 0,00 & 1,24 & 0,00 & 0,00 & 1,11 & 2,14 & 0,00 \\
\hline CUYABENO & 0,00 & 0,00 & 2,65 & 0,00 & 1,24 & 2,39 & 0,00 \\
\hline GONZALO PIZARRO & 0,00 & 1,32 & 0,00 & 0,00 & 0,00 & 0,00 & 1,10 \\
\hline LAGO AGRIO & 2,70 & 2,58 & 3,26 & 1,89 & 2,81 & 2,12 & 1,71 \\
\hline PUTUMAYO & 3,11 & 0,00 & 1,43 & 0,00 & 1,33 & 1,29 & 1,25 \\
\hline SHUSHUFINDI & 0,60 & 0,29 & 1,10 & 0,79 & 1,28 & 0,74 & 0,96 \\
\hline SUCUMBIOS & 0,00 & 6,48 & 0,00 & 0,00 & 2,90 & 0,00 & 2,71 \\
\hline
\end{tabular}

Fuente: SOLCA

Elaboración: Autores.
La segunda tasa más alta de incidencia acumulada en el período de estudio es en la provincia de Napo, cuya tendencia se aprecia a continuación:

\section{GRÁFICO \# 5}

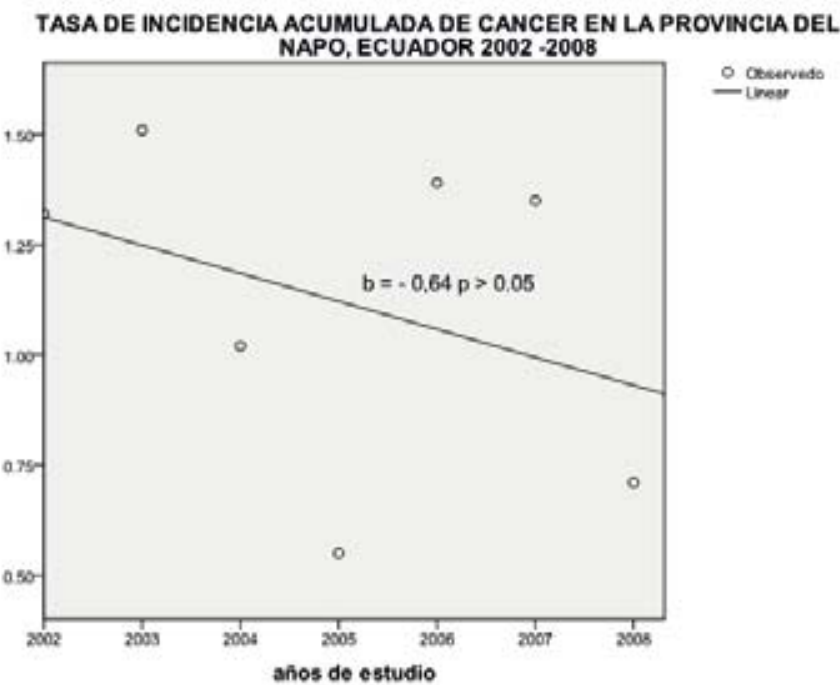

Fuente: SOLCA

Elaboración: Autores

Nuevamente se puede apreciar que al momento no es posible establecer una tendencia de la tasa de incidencia acumulada en esta provincia.

La distribución del cáncer según los cantones que conforman esta provincia se aprecia en la siguiente tabla: 
TABLA \# 4

TASA DE INCIDENCIA ACUMULADA DE CÁNCER EN LOS CANTONES DE LA PROVINCIA DE NAPO, ECUADOR 2002 - 2008

\begin{tabular}{|l|ccccccc|}
\hline CANTONES & $\mathbf{2 0 0 2}$ & $\mathbf{2 0 0 3}$ & $\mathbf{2 0 0 4}$ & $\mathbf{2 0 0 5}$ & $\mathbf{2 0 0 6}$ & $\mathbf{2 0 0 7}$ & $\mathbf{2 0 0 8}$ \\
\hline NAPO & 1.3 & 1.5 & 1.01 & 0.54 & 1.39 & 1.53 & 0.71 \\
\hline \hline ARCHIDONA & 0.5 & 0.49 & 0.96 & 0.46 & 0 & 0.44 & 0 \\
\hline C. AROSEMENA & 0 & 0 & 0 & 0 & 2.88 & 0 & 0 \\
\hline EL CHACO & 4.6 & 0 & 1.45 & 0 & 1.38 & 1.34 & 0 \\
\hline QUIJOS & 5.1 & 3.34 & 1.62 & 0 & 7.7 & 1.49 & 0 \\
\hline TENA & 0.8 & 1.8 & 0.97 & 0.75 & 1.105 & 1.79 & 1.22 \\
\hline
\end{tabular}

Fuente: SOLCA

Elaboración: Autores.

Similar tendencia se observa en las restantes provincias que comparativamente con las dos anteriores podrían considerarse como de intermedia tasa de incidencia acumulada como son Pastaza y Orellana.

\section{GRÁFICO \# 6}

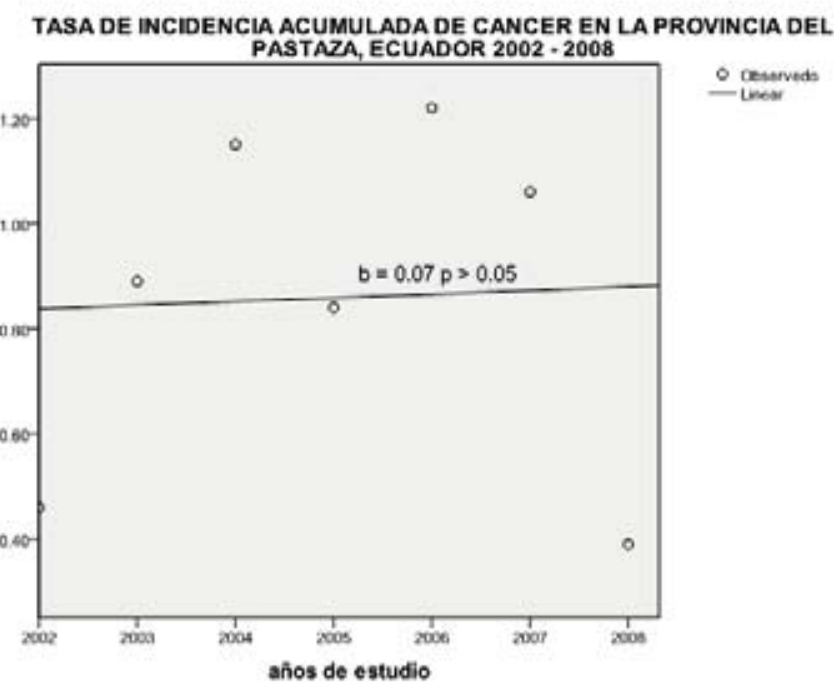

Sibien en esta provincia aparece una tendencia al crecimiento de la tasa de incidencia acumulada, ésta no es significativa.

Algo similar ocurre en la provincia de Orellana, en la que tampoco se evidencia un decrecimiento significativo de la tasa de incidencia acumulada.

\section{GRÁFICO \# 7}

\section{TASA DE INCIDENCIA ACUMULADA DE CANCER EN LA PROVINCIA DE} ORELLANA, ECUADOR $2002-2008$

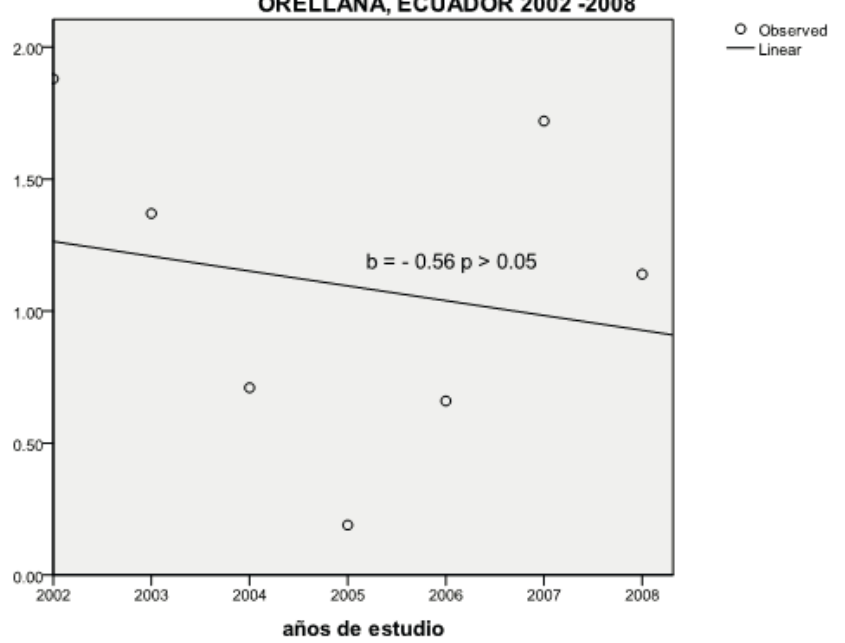

Elaboración: Autores.

La tendencia observada en los cantones de la provincia del Pastaza se presenta a continuación:

Fuente: SOLCA

Elaboración: Autores 
TABLA \# 5

TASA DE INCIDENCIA ACUMULADA DE CÁNCER EN LOS CANTONES DE LA PROVINCIA DE PASTAZA, ECUADOR 2002 - 2008

\begin{tabular}{|cccccccc|}
\hline CANTONES & $\mathbf{2 0 0 2}$ & $\mathbf{2 0 0 3}$ & $\mathbf{2 0 0 4}$ & $\mathbf{2 0 0 5}$ & $\mathbf{2 0 0 6}$ & $\mathbf{2 0 0 7}$ & $\mathbf{2 0 0 8}$ \\
\hline PASTAZA & 0,46 & 0.89 & 1,15 & 0,83 & 1,22 & 1,05 & 0,38 \\
\hline ARAJUNO & 0 & 0 & 0 & 1,67 & 0 & 0 & 0 \\
\hline MERA & 0 & 0 & 1,09 & 1,06 & 1,03 & 2,01 & 0,98 \\
\hline PASTAZA & 0,62 & 1,21 & 1,36 & 0,75 & 1,47 & 1,07 & 0,34 \\
\hline
\end{tabular}

Fuente: SOLCA

Elaboración: Autores

Esta misma distribución se obtuvo para la provincia de Orellana:

\section{GRÁFICO \# 8}

TASA DE INCIDENCIA ACUMULADA EN LOS CANTONES DE LA PRO-

VINCIA DE ORELLANA, ECUADOR 2002 - 2008

TASA DE INCIDENCIA ACUMULADA DE CANCER EN LA PROVINCIA DE

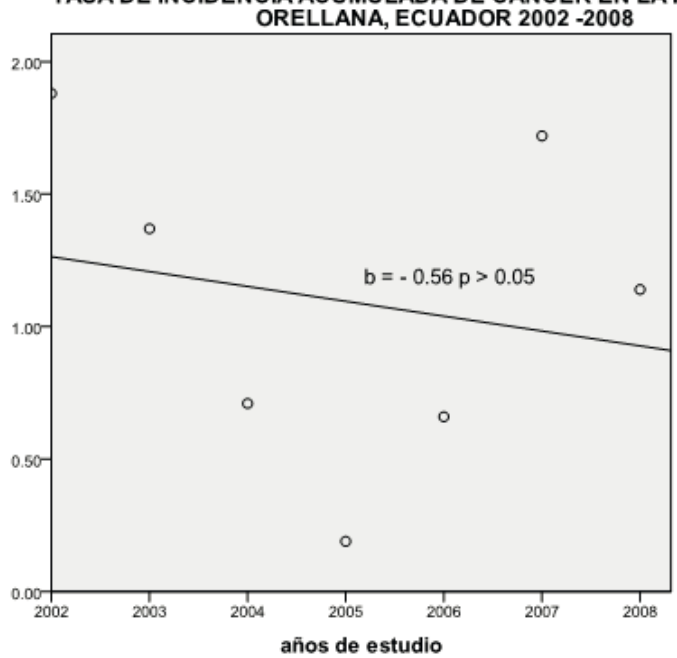

Fuente: SOLCA

Elaboración: Autores.

La situación de la tendencia de esta tasa en sus cantones es la siguiente:
TABLA \# 6

TASA DE INCIDENCIA ACUMULADA DE CÁNCER POR CANTONES EN LA PROVINCIA DE ORELLANA, ECUADOR $2002-2008$

\begin{tabular}{|c|c|c|c|c|c|c|c|c|}
\hline CANTONES & $\mathbf{2 0 0 2}$ & $\mathbf{2 0 0 3}$ & $\mathbf{2 0 0 4}$ & $\mathbf{2 0 0 5}$ & $\mathbf{2 0 0 6}$ & $\mathbf{2 0 0 7}$ & $\mathbf{2 0 0 8}$ \\
\hline ORELLANA & 1,87 & 1,36 & 0,7 & 0,19 & 0,65 & 1,71 & 1,13 \\
\hline \hline AGUARICO & 0 & 3,91 & 0 & 0 & 0 & 0 & 0 \\
\hline LA JOYA DE & 2,17 & 1,03 & 0,33 & 0,31 & 0,92 & 0,59 & 0,57 \\
\hline LOS SACHAS & 2,17 & 0,67 & 0 & 0 & 0 & 0 & 0 \\
\hline LORETO & 0,7 & $0,1,15$ & 1,24 & 0 & 0,77 & 3,15 & 1,43 \\
\hline ORELLANA & 1,81 & 1,51 &
\end{tabular}

Fuente: SOLCA

Elaboración: Autores

En el gráfico número 9 se observa la tendencia de la tasa de incidencia acumulada en la provincia de Morona Santiago:

\section{GRÁFICO \# 9}

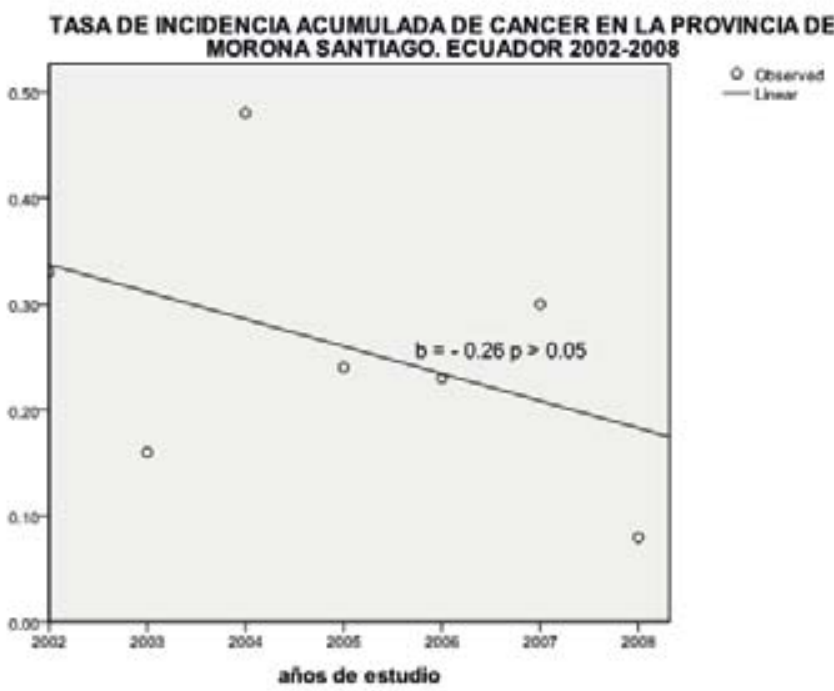

Fuente: SOLCA

Elaboración: Autores

La distribución de casos de cáncer y /o tumores malignos por cantones al interior de esta provincia es la siguiente: 
TABLA \# 7

TASA DE INCIDENCIA ACUMULADA DE CÁNCER POR CANTONES EN LA PROVINCIA DE MORONA SANTIAGO, ECUADOR $2002-2008$

\begin{tabular}{|c|c|c|c|c|c|c|c|}
\hline CANTONES & $\mathbf{2 0 0 2}$ & $\mathbf{2 0 0 3}$ & $\mathbf{2 0 0 4}$ & $\mathbf{2 0 0 5}$ & $\mathbf{2 0 0 6}$ & $\mathbf{2 0 0 7}$ & $\mathbf{2 0 0 8}$ \\
\hline Morona & $\mathbf{0 , 3 3}$ & $\mathbf{0 , 1 6}$ & $\mathbf{0 , 4 8}$ & $\mathbf{0 , 2 4}$ & $\mathbf{0 , 2 3}$ & $\mathbf{0 , 3 0}$ & $\mathbf{0 , 0 8}$ \\
\hline MORONA & 0,63 & 0,00 & 1,23 & 0,61 & 0,90 & 0,89 & 0,00 \\
\hline PALORA & 1,50 & 1,48 & 0,00 & 0,00 & 0,00 & 0,00 & 0,00 \\
\hline SUCUA & 0,66 & 0,00 & 6,39 & 0,63 & 0,00 & 0,00 & 0,00 \\
\hline THAISHA & 0,00 & 0,00 & 0,72 & 0,00 & 0,00 & 0,00 & 0,68 \\
\hline
\end{tabular}

Fuente: SOLCA

Elaboración: Autores

Igual que en Morona, la tendencia en la provincia de Zamora, tampoco logró demostrar una tendencia que pueda considerarse significativa.

\section{GRÁFICO \# 10}

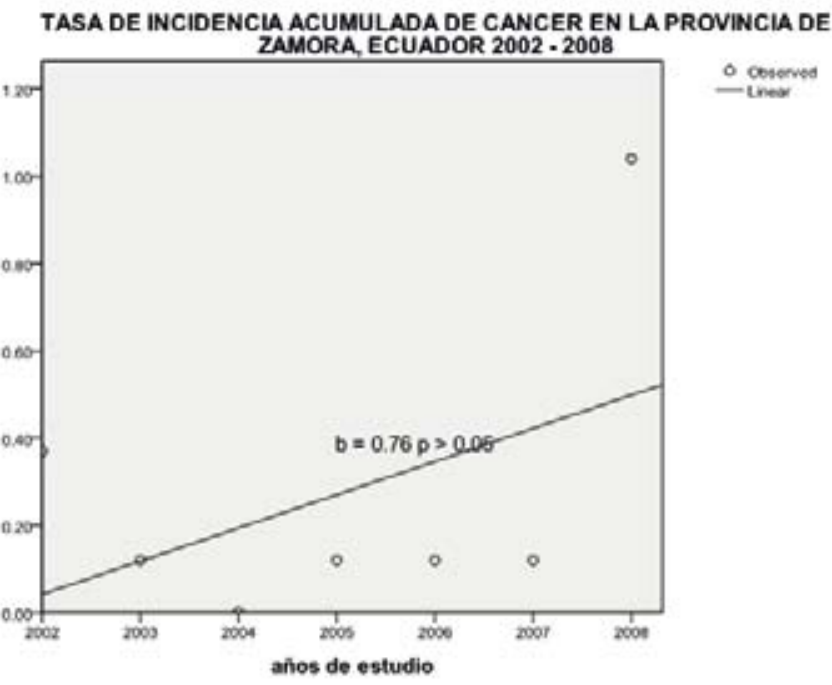

Elaboración: Autores.

En los cantones de la provincia de Zamora, la tendencia de la tasa de incidencia acumulada de cáncer a lo largo del período estudiado fue:
TABLA \# 8

TASA DE INCIDENCIA ACUMULADA DE CÁNCER EN LOS CANTONES DE LA PROVINCIA DE ZAMORA CHINCHIPE, ECUADOR 2002 -2008

\begin{tabular}{|l|ccccccc|}
\hline CANTONES & 2002 & $\mathbf{2 0 0 3}$ & $\mathbf{2 0 0 4}$ & $\mathbf{2 0 0 5}$ & $\mathbf{2 0 0 6}$ & $\mathbf{2 0 0 7}$ & $\mathbf{2 0 0 8}$ \\
\hline $\begin{array}{l}\text { ZAMORA } \\
\text { CHINCHIPE }\end{array}$ & 0,24 & 0,36 & 0,12 & 0 & 0,11 & 0,11 & 0,11 \\
\hline $\begin{array}{c}\text { CENTINELA } \\
\text { DEL CONDOR }\end{array}$ & 0 & 1,68 & 0 & 0 & 0 & 0 & 0 \\
\hline CHINCHIPE & 1,11 & 1,1 & 0 & 0 & 0 & 0 & 0 \\
\hline EL PANGUI & 0 & 0 & 0 & 0 & 0 & 1,2 & 0 \\
\hline YANTZAZA & 0 & 0,64 & 0 & 0 & 0,62 & 0 & 0 \\
\hline ZAMORA & 0,43 & 0 & 0,42 & 0 & 0 & 0 & 0,4 \\
\hline
\end{tabular}

Fuente: SOLCA

Elaboración: Autores.

Algo similar ocurre en cuanto a la tendencia de esta tasa en la provincia de Morona Santiago.

Finalmente, en las dos provincias que comparativamente tienen tasas de incidencia acumulada de cáncer baja como son Zamora Chinchipe y Morona Santiago, la tendencia de estas tasas tampoco demostró significancia estadística:

La edad al diagnóstico de los casos de cáncer varía en las provincias, lo cual puede en alguna medida estar relacionado con la oportunidad del diagnóstico tanto por sospecha personal como por sospecha médica. En la siguiente tabla se presenta esta información: 
TABLA \# 9

PROMEDIO DE EDAD DEL DIAGNÓSTICO DE CÁNCER DE PACIENTES PROVENIENTES DE PROVINCIAS DEL ORIENTE ECUATORIANO

\begin{tabular}{|l|c|c|c|c|c|c|c|c|}
\hline Provincia & Promedio & M ediana & $\begin{array}{c}\text { Desviac. } \\
\text { Típica }\end{array}$ & $\begin{array}{c}\text { Percentil } \\
\mathbf{2 5}\end{array}$ & $\begin{array}{c}\text { Percentil } \\
\mathbf{5 0}\end{array}$ & $\begin{array}{c}\text { Percentil } \\
\mathbf{7 5}\end{array}$ & $\begin{array}{c}\text { Edad } \\
\text { M ínima }\end{array}$ & $\begin{array}{c}\text { Edad } \\
\text { Máxima }\end{array}$ \\
\hline Morona & 49 & 55 & 23,6 & 32 & 55 & 70 & 2 & 88 \\
\hline Napo & 51,5 & 52 & 16,9 & 39,5 & 52 & 64 & 3 & 87 \\
\hline Pastaza & 49 & 50 & 18,5 & 35 & 50 & 63 & 8 & 86 \\
\hline Zamora & 56 & 61 & 18,9 & 50,5 & 61 & 68 & 10 & 72 \\
\hline Sucumbíos & 48,9 & 48 & 16,2 & 38 & 48 & 60 & 3 & 84 \\
\hline
\end{tabular}

Fuente: SOLCA

Elaboración: Autores.

Si bien, comparado los promedios de edad al diagnóstico en las provincias en donde se concentran las empresas petroleras hay un promedio de edad menor al momento de la confirmación de la neoplasia o tumor, no se encontraron diferencias significativas al realizar el análisis ANOVA.

Se mencionó previamente que la principal localización en mujeres corresponde al cérvix codificado como 593. El promedio de edad de las mujeres que lo padecen fue de 47,6 años, con una mediana de 47 años y una distribución multimodal, siendo su desviación estándar de 13,9 años. En 25\% de las mujeres son diagnosticadas antes de los 23 años, y el 75\% antes de los 57 años. La edad mínima al momento del diagnóstico fue de 21 años y la máxima edad de 84 años.

Esta información desagregada de acuerdo a las principales provincias que lo registran se observa a continuación:
TABLA 10

PROMEDIO DE EDAD DEL DIAGNÓSTICO DE CÁNCER DE CERVIX DE PACIENTES PROVENIENTES DE PROVINCIAS DEL ORIENTE ECUATORIANO

\begin{tabular}{|l|c|c|c|c|c|c|c|c|}
\hline Provincia & Promedio & M ediana & $\begin{array}{c}\text { Desviac. } \\
\text { Típica }\end{array}$ & $\begin{array}{c}\text { Percentil } \\
\mathbf{2 5}\end{array}$ & $\begin{array}{c}\text { Percentil } \\
\mathbf{5 0}\end{array}$ & $\begin{array}{c}\text { Percentil } \\
\mathbf{7 5}\end{array}$ & M ínima & Máxima \\
\hline Morona & 53,5 & 53,5 & 27,27 & 34 & 53,5 & $\mathbf{7 3}$ & 39 & 75 \\
\hline Napo & 48,7 & 48 & 13,7 & 40 & 48 & 57,5 & 21 & 83 \\
\hline Pastaza & 48,3 & 46 & 16,6 & 33,5 & 46 & 67 & 28 & 71 \\
\hline Zamora & 60 & 60 & & 36,5 & 60 & 60 & 60 & 60 \\
\hline Sucumbíos & 46,7 & 47 & 12,73 & 36,5 & 47 & 44 & 22 & 78 \\
\hline
\end{tabular}

Fuente: SOLCA

Elaboración: Autores.

Algo similar se observa en el caso de los varones, en quienes el cáncer relacionado con estómago que corresponde a las codificaciones 159-160-162-163-184-165-168 y 169 fue el que mayor tasa de incidencia acumulada se observó. El promedio de edad de diagnóstico en varones de este cáncer fue de 58,25 años, con una mediana y moda de 59 años y una desviación estándar de 7,69 años. El percentil 25 de la edad de diagnóstico fue de 53,25 años y el percentil 75 fue de 64,5 años. La mínima edad de diagnóstico encontrada fue de 39 años y la edad más alta de 69 años.

En la provincia de Sucumbíos fue en donde en forma casi exclusiva este cáncer se presentó:

TABLA \# 11

PROMEDIO DE EDAD DEL DIAGNÓSTICO DE CÁNCER DE ESTÓMAGO DE LOS PACIENTES PROVENIENTES DE PROVINCIAS DEL ORIENTE ECUATORIANO

\begin{tabular}{|l|c|c|c|c|c|c|c|c|}
\hline Provincia & Promedio & M ediana & $\begin{array}{c}\text { Desviac. } \\
\text { Típica }\end{array}$ & $\begin{array}{c}\text { Percentil } \\
\mathbf{2 5}\end{array}$ & $\begin{array}{c}\text { Percentil } \\
\mathbf{5 0}\end{array}$ & $\begin{array}{c}\text { Percentil } \\
\mathbf{7 5}\end{array}$ & M ínima & Máxima \\
\hline Morona & 55,5 & 55,5 & 6,3 & 31,5 & 47,9 & 66,8 & 50,8 & 67 \\
\hline Napo & 62,5 & 62 & 9,6 & 57,7 & 65,9 & 75,7 & 54,4 & 77,6 \\
\hline Pastaza & 57,3 & 61 & 11,9 & 57,5 & 65,9 & 67,4 & 49 & 65,9 \\
\hline Zamora & 61 & 61 & & 61 & 61 & 61 & 61 & 61 \\
\hline Sucumbíos & 57,6 & 59 & 8,39 & 52,5 & 59 & 63,5 & 39 & 69 \\
\hline
\end{tabular}

Fuente: SOLCA

Elaboración: Autores 
Se puede evidenciar que la mediana de edad al momento del diagnóstico es menor en las provincias con predominio petrolero, pero pone en evidencia también el diagnóstico a edades adultas.

\section{EL PETRÓLEO Y EL CÁNCER}

Si bien rebasa el objetivo de este estudio el demostrar o no la relación cáncer en esta zona con la presencia de empresas petroleras, se encontró una relación no aleatoria en la distribución de la tasa de incidencia acumulada de cáncer en las provincias.

En el siguiente mapa se puede apreciar la gran variedad de empresas que se dedican a la actividad petrolera, que estuvieron presentes hasta el año 2008.

\section{GRÁFICO \# 3}

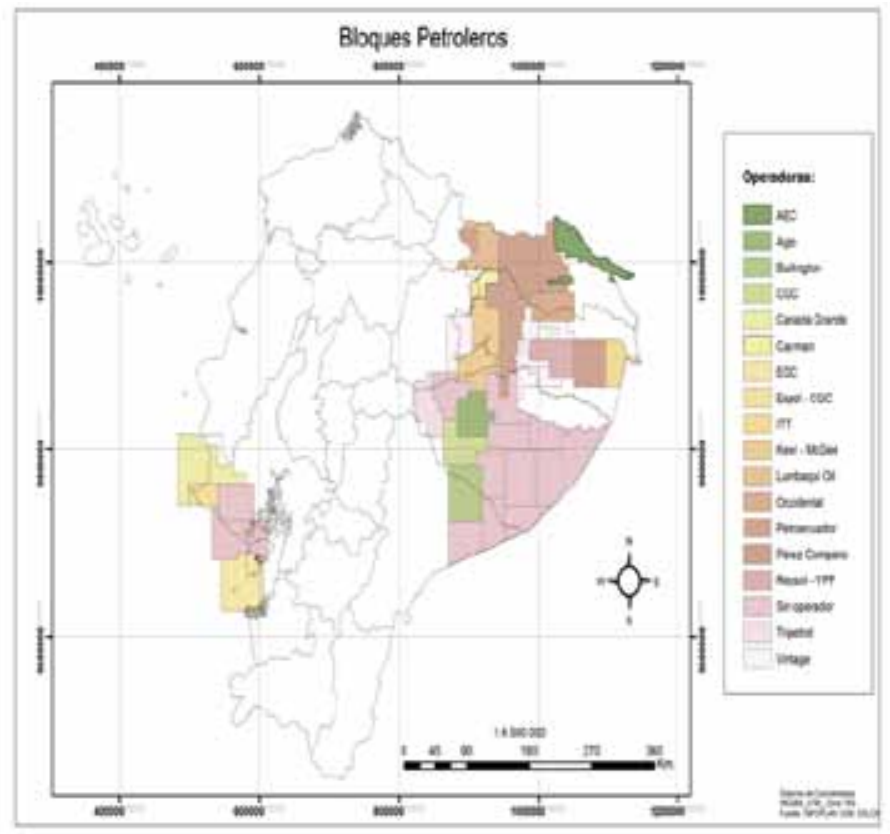

Las zonas donde se encuentran la distribución de la tasa de incidencia acumulada por cáncer y/o tumores en las zonas donde se encuentran las empresas que se dedican bien sea a la exploración o a la explotación del petróleo se pueden apreciar en el siguiente mapa:

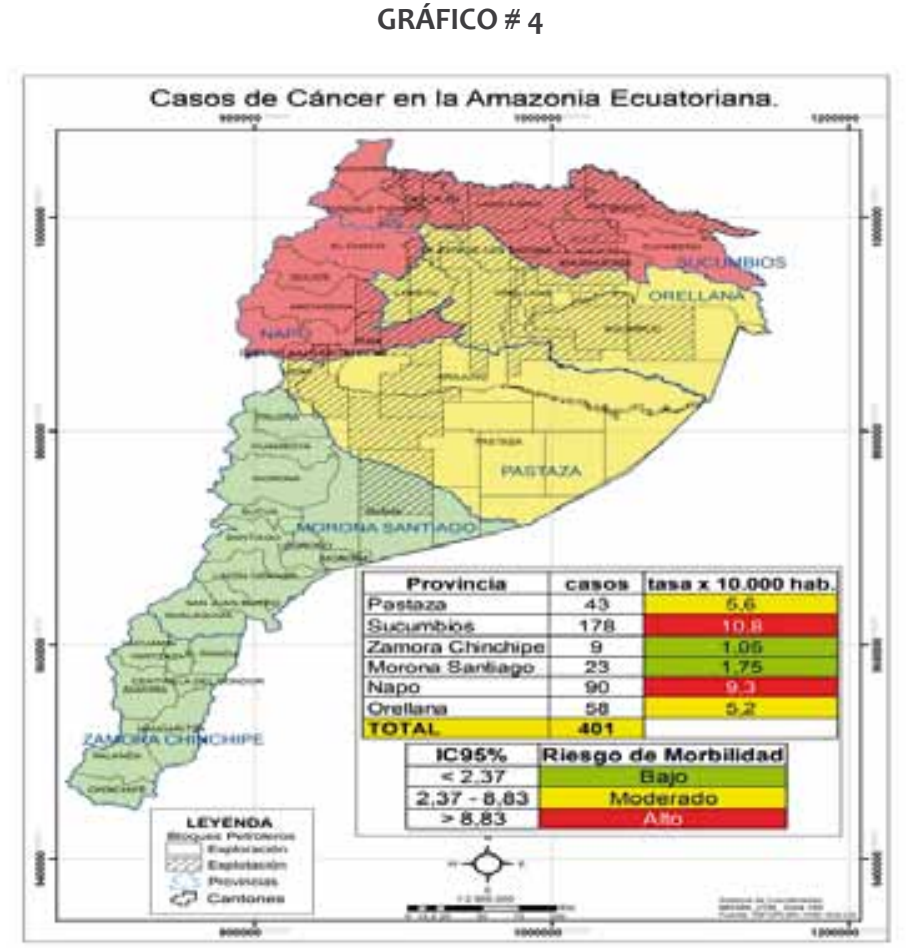

Para el análisis se consideró a las provincias según la tasa de incidencia acumulada de cáncer en tres zonas, denominando como riesgo bajo, moderado o alto. Esto se realizó considerando los intervalos de confianza de las tasas de incidencia acumulada provinciales.

Las diferencias observadas entre las tasas de incidencia acumulada en las provincias objeto de estudio, permitió la realización de una función de autocorrelación espacial para el año 2008, aplicando la corrección de Morán, para establecer la aleatoriedad o no de la distribución espacial de las tasas de incidencia acumulada entre las diversas provincias, con lo que se podría evidenciar finalmente si la subdivisión en provincias con mayor o menor tasa de incidencia acumulada podría no ser producto del azar.

Los cálculos realizados demostraron un Índice I igual a 1,92, con un valor esperado del Índice I, esto es un EI igual -0,2, lo que determinó un puntaje $z=2,07$, superior al esperado para el 95\% de certeza, concluyéndose que las diferencias observadas son 
estadísticamente significativas, es decir que la distribución provincial de casos de cáncer y/o tumores observada, al parecer no sería aleatoria; o dicho de otra forma, podría considerarse la posibilidad de que en aquellas provincias donde la tasa de incidencia acumulada es mayor, las mismas que corresponden a aquellas con mayor actividad petrolera, se distribuyen mayoritariamente los casos de cáncer.

\section{DISCUSIÓN}

Existen muchas teorías sobre el cáncer y asociaciones que se dan con el petróleo y sus derivados, siendo varios los intentos por involucrar en forma causal diversos eventos como cáncer y petróleo. Hay una realidad objetiva que es el padecimiento de cáncer de diversa localización, en los pacientes de la Amazonia y lo que se pretendió es crear un perfil de la localización de los casos de cáncer en las diversas provincias del Oriente ecuatoriano en las que se explota el petróleo.

Seguramente muchas variables intervienen en la presencia de cáncer, como son aspectos genéticos, oportunidad de diagnóstico que son difíciles de controlar o al menos de establecer. Se necesita saber en promedio cuanto dura la enfermedad del cáncer y es necesario segmentar en percentiles la edad para saber cuanto influye esta variable en el aparecimiento y desarrollo de la enfermedad. La mediana y promedio de edad fue de 50 y 51 años en este estudio, es decir población adulta con buena expectativa de vida en relación a la esperanza de vida en el Ecuador que es de 75 años.

La primera causa encontrada de cáncer en varones corresponde al tracto gastrointestinal. Hay que recordar que los habitantes del Oriente son colonos de diferentes provincias sin que se haya determinado a profundidad la prevalencia de los factores de riesgo que puede predisponer a estos diferentes tipos de cáncer en cada provincia. En diversos estudios se da cuenta del grado diferencial de la incidencia de este cáncer, así Hussein NR en su artículo Helicobacter pylori and gastric cancer in the Middle East: a new enigma? (7) establece que una carga genética podría predisponer a padecer cáncer ante otro tipo de factores de riesgo, estableciendo la multi etiología de la enfermedad.

La revisión de la literatura científica da cuenta de la gran evidencia que han aportado las investigaciones que tratan de establecer a nivel genético y molecular la posible causa de la alteración que lleva al cáncer; sin embargo, se hace necesario el estudio de las condiciones que favorecen o limitan el aparecimiento del cáncer, así como las condiciones medioambientales.

Otros estudios dan cuenta también de las relaciones existentes con el tabaco, medicamentos, etc. (8) pero las condiciones de vida de las personas que viven en el Oriente, no brindan la oportunidad de tener acceso siquiera a información, o a medidas de prevención o diagnóstico precoz, siendo estas variables sin duda de gran importancia para el desarrollo del Cáncer.

Por otra parte están los factores y patrones alimenticios que tienen una gran variación geográfica con base a la oportunidad de siembra y autoconsumo, (9). Pero dónde están los servicios de salud?, cuáles son las medidas tendientes a determinar con exactitud la real dimensión de este problema en el Oriente ecuatoriano y cuáles son las medidas tendientes a mitigar esta problemática?. Seguramente hay sinergismo entre los diferentes factores que producen el cáncer.

En el presente estudio la función de correlación espacial ayudó a determinar si las diferentes tasas de incidencia acumuladas por cáncer y/o tumores observadas en estos años, en cada una de las provincias del Oriente ecuatoriano tienen una distribución aleatoria, lo cual dejó ver que sin que se pretenda establecer causalidad, al ser mayor estas tasas en las provincias en donde mayor actividad petrolera existe, también hubo mayor incidencia de casos de cáncer de diversa etiología, lo que sugiere una relación entre estas variables.

Sería poco probable encontrar estas tasas de incidencia acumulada por neoplasias en una población con una densidad 
poblacional estimada para el año 2008 de 0,25 habitantes por kilómetro cuadrado, sin descartarse obviamente alteraciones endogámicas.

Un tema especial corresponde al papel de los servicios de salud, no parece existir una idea clara de la edad a la que se presentan con mayor frecuencia los procesos neoplásicos en el Ecuador; sin embargo, una quinta parte de los pacientes atendidos han fallecido. Por la edad de diagnóstico primario daría la impresión en términos absolutamente generales que los diagnósticos son tardíos, lo cual puede ser debido a varias razones como la inexistencia de servicios de salud especializados en oncología en todo el Oriente ecuatoriano que permitan detectar a tiempo este problema, la falta de educación que sobre este tema que todos los ecuatorianos eventualmente debemos tener, al no poder diferenciar signos o síntomas tempranos del cáncer, situación particularmente difícil por la amplia gama de manifestaciones clínicas, muchas de ellas silentes.

A esto bien podría sumarse la capacidad económica de la gran mayoría de los pobladores que seguramente requiere de dinero en efectivo hasta para desplazarse a los centros de diagnóstico, que si bien brindan en todos los casos una ayuda importante, el mismo desplazamiento hacia estos centros obliga a dejar sus tierras y por tanto la producción de la que posiblemente muchos de los casos diagnosticados de cáncer de la zona del Oriente son su fuente de vida.

Es poco verosímil la tendencia observada en todas las provincias analizadas, situación que rebasa los alcances de este estudio oncológico, pero un decrecimiento de la tasa de incidencia, si bien en ningún caso es significativo, puede estar influenciada por situaciones como emigración de los casos o sospechosos a otras ciudades para diagnóstico. Así, mayoritariamente acuden a Quito, personas provenientes de Sucumbíos, por la cercanía; sin embargo, es posible que a centros oncológicos situados en Ambato, Cuenca o inclusive Guayaquil, sea conveniente acudir a aquellos pacientes residentes en provincias cercanas a estas ciudades.
A esto se suma el hecho de que analizando los datos, son las cabeceras provinciales, los sitios en donde se tiene el mayor número de casos, lo cual resulta previsible al encontrarse en estas capitales de provincia los centros hospitalarios de mayor complejidad y capacidad resolutiva a pesar de no disponer de especialistas oncólogos.

Este hecho se intentó controlar con la georeferenciación a nivel de terreno de la dirección domiciliaria (cantón) de los casos de cáncer diagnosticados que residían al menos 5 años en esa dirección, sin embargo, se evidenció errores en la información que los pacientes proporcionan para los registros oncológicos, en donde se consigna la dirección actual del paciente, sin tomarse en cuenta que muchos pacientes indican la dirección de un familiar o del sitio al cual llegan en Quito para poder tener facilidades y trasladarse al hospital oncológico.

\section{CONCLUSIONES}

1.- La información que consta en el Registro Nacional de Tumores es básica que se limita a características sociodemográficas y que no permite realizar un análisis más profundo en la búsqueda de posibles factores de asociación/causalidad entre las diferentes variables.

2.- Las provincias con las tasas más altas de riesgo de cáncer encontradas fueron $\mathrm{Napo}$ (9.3) y Sucumbíos (10.8). Las provincias con riesgo intermedio de riesgo de cáncer fueron Pastaza (5.6) y Orellana (5.2), y las de más bajo riesgo fueron Morona Santiago (1.75) y Zamora Chinchipe (1.05).

3.- El mayor porcentaje de casos de cáncer detectados fueron Cáncer del Tracto Genito-urinario femenino (34\%), seguido de Cáncer gastro-intestinal (16,9\%), Cáncer de Piel y Relacionados (12,4\%) entre los de mayor incidencia acumulada. 
4.- Con respecto al género masculino los porcentajes más altos de cáncer detectados fueron Tracto Gastro-intestinal (28,1\%), seguido de Cáncer de Piel y Relacionados (18,5\%), Cáncer del Aparato Genital Masculino (17,8\%) y Cáncer del Sistema Hematopoyético $(14,4 \%)$ entre los de mayor frecuencia.

5.- Con respecto al género femenino el Cáncer relacionado al Aparato Genitourinario fue el de mayor porcentaje de casos (53,3\%), Cáncer de la Glándula Mamaria (11,7\%) y Cáncer de Piel y Relacionados $(8,9 \%)$ entre los de más alta frecuencia.

\section{BIBLIOGRAFIA}

Álava, P. (2006). Dinámica de la deforestación en el cantón La Joya de Los Sachas. Tesis de Ingeniería. Dep. Geografía. PUCE. Quito.

Angelsen, A. (1999). Agricultural Expansion and Deforestation: Modeling the Impact of Population, Market forces and Poverty Rights. En: Journal of Development Economics. 58 (pp. 155 -218).

Barrantes, G. Chaves, H. y Vinueza, M. (2001). El Bosque en el Ecuador. Una Visión Transformadora para el Derecho y la Conservación. Quito. COMAFORS (Corporación de manejo forestal sustentable).

Figueroa JD et al.(2009). Cigarette smoking, body mass index, gastro-esophageal reflux disease, and non-steroidal antiinflammatory drug use and risk of subtypes of esophageal and gastric cancers by P53 overexpression. Cancer Causes Control; 20(3): 361-8.

Glass D, Adams G., Manuell R., Bisby J. (2000). RETROSPECTIVE EXPOSURE ASSESSMENT FOR BENZENE IN THE AUSTRALIAN PETROLEUM INDUSTRY Ann. occup. Hyg., Vol. 44, No. 4, pp. 301;320.

G K Raabe and O Wong. (1996). LEUKEMIA MORTALITY BY CELL TYPE IN PETROLEUM WORKERS WITH POTENTIAL EXPOSURE TO BENZENE. EN: Environ Health Perspect. 1996 December; 104(Suppl 6): 1381-1392.
INFORME YANA CURI: IMPACTO DE LA ACTIVIDAD PETROLERA EN POBLACIONES RURALES DE LA AMAZONIA ECUATORIANA. Instituto de Epidemiologia y Salud Comunitaria “Manuel Amunarriz". Coca. 2002.

Helicobacter pylori and gastric cancer in the Middle East: ¿a new enigma? Hussein NR; World J Gastroenterol; 16(26): 3226-34. $2010 \mathrm{Jul} 14$.

Hurting Anna-Karin and Miguel San Sebastián (2002). Geographical differences in cancer incidence in the Amazon basin of Ecuador in relation to residence near oil fields. En: International Journal of Epidemiology 2002;31:1021-1027

Kimerling, J. (1993). Crudo Amazónico. Ed: ABYA-YALA. Quito.

Martínez, C. y Sánchez, D. (2004) Propuesta de Modelo para la Identificación de las Áreas de Manejo Especial de la Reserva Biológica de Limoncocha. ESPE. Quito-Ecuador.

Martyn T. Smith, Rachael M. Jones, and Allan H. Smith. (March 2007). BENZENE EXPOSURE AND RISK OF NON-HODGKIN LYMPHOMA. en: Cancer Epidemiol Biomarkers Prev 2007; 16(3).

Noboa, R. (2007). Estudio de Dinámica Poblacional y Fragmentación del Bosque Natural en la Provincia de Orellana entre los años 1986 - 2002. Tesis de Ingeniería Dep. Geografía. PUCE. Quito.

Review of salt consumption and stomach cancer risk: epidemiological and biological evidence. Wang XQ et al. World J Gastroenterol; 15(18): 2204-13, 2009 May 14.

San Sebastián M., B Armstrong, J A Córdoba, C Stephens, (2001). Exposures and cancer incidence near oil fields in the Amazon basin of Ecuador. En: Occup Environ Med; 58:517-522

SENPLADES-INFOPLAN. (2006). Secretaría Nacional de Planificación y Desarrollo. Plan Nacional de Desarrollo

Sierra, R. (2000). Dynamics and Patterns of Deforestation in Western Amazon. En: Applied Geography. 20 (pp.1-16).

Smith Martyn T., Rachael M. Jones, and Allan H. Smith. (March 2007). BENZENE EXPOSURE AND RISK OF NON-HODGKIN LYMPHOMA. En: Cancer Epidemiol Biomarkers Prev 2007;16(3). 
